\title{
Systematic on fission fragment mass distribution of ${ }^{235} \mathrm{U}$ fission
}

\author{
Liu Tingjin, Sun Zhengjun, Shu Nengchuan, and Liu Ping \\ China Nuclear Data Center, China Institute of Atomic Energy, Beijing, P.R. China
}

\begin{abstract}
Based on the fission fragment mass distribution data up to $20 \mathrm{MeV}$ measured by Hambsch with double kinetic energy method (KEM) and other chain yield data measured with radio active method (RAM), the systematic of fission fragment mass distribution was investigated by using 5 Gaussian model. A code of nonlinear least squares method was developed and the parameters, called discrete parameters, were deduced by fitting the experimental data at each energy point measured. The discrete parameters as functions of energy were fitted with second order functions by least squares method, so that the parameters, called systematic parameters, can be calculated at any energy point. With the systematic, the yields of any mass A at any energy in the region from 0 to $20 \mathrm{MeV}$ can be calculated. The calculated results could reproduce the experimental data measured with KEM well, but show somewhat systematical difference from the data measured by RAM, which reflects there is some systematical difference between the two kinds of measured data. The possible reason is that the data measured by KEM need more complicated accurate corrections.
\end{abstract}

\section{Introduction}

The energy dependence of the fission fragment mass distribution is very important in the field of fission physics and fission yield study for both science and practical application. It is going on in three ways at present: experimental measurement, theoretical calculation and systematic. Systematic is the most simple and hopeful, if there are enough experimental data as a base. Hambsch's data are very useful for this purpose.

\section{Data base}

The following experimental fragment mass distribution or chain yield data were used as the base of the systematic: F.J. Hambsch [1] in the energy region from 0.5 to $6.0 \mathrm{MeV}$ by 0.5 MeV step; R.B. Strittmatter [2], G. Diiorio [3], G. Siegert [4] at thermal energy; W.J. Maeck [5] at $0.4 \mathrm{MeV}$; J.W. Mandler [6] at 9.0, 15.0 MeV; Bao Jie [7] at $19.1 \mathrm{MeV}$ and Feng Jing [8] at $22.0 \mathrm{MeV}$.

Among them, Hambsch's data were measured by using double kinetic energy method (KEM), with which the fragment mass distribution data can be measured at one time for more complete mass number A. So the systematical error can be avoided for different mass number A. But the data need to be corrected for mass (energy) resolution, energy losses, neutron emission and others, which are quite complicated. The other data were measured by using radio active method (RAM), with which the data measured are chain yield, and the radio decay, $\gamma$ or $\beta$, of fission product nuclides are determined for each product nuclide. RAM is difficult to measure for more complete mass number $\mathrm{A}$, but there is no problem of mass resolution correction.

Exactly fragment mass distribution is different from the chain yield due to the fact there exist delayed neutron emission, but the portion of the delayed neutron emission is, in general, quite small, so for our purpose they can be think approximately the same.
J.W. Mandler's data are the ratio of chain yield to the mass chain $\mathrm{A}=140$, the data were calculated into chain by using standard chain yield for $A=140$ evaluated by ourselves [9].

All data were smoothed [10] for each 9 points before they were used for the systematic calculation. Otherwise, it would be difficult to fit, especially for the data measured by radio active method.

\section{Multi Gaussian model on mass distribution}

1) Mass distribution data can be fitted with 5 Gaussian model:

$$
Y(A)=\sum_{i=1}^{I} y_{i} / \sqrt{2 \pi} \sigma_{i} \times \exp \left(-\left(A-a+\Delta_{i}\right)^{2} / 2 \sigma_{i}^{2}\right.
$$

where $\boldsymbol{I}=5$, and

$$
a=\left(A_{F}-v\right) / 2 \text {. }
$$

$A_{F}$ is the mass of fission system and $v$ is prompt $v$-value of the fission nuclide. Due to the symmetry of the distribution, there are altogether 9 adjusted parameters: $\Delta_{1}, \Delta_{2}, y_{1}, y_{2}, y_{3}, \sigma_{1}, \sigma_{2}$, $\sigma_{3}, a$, where $\Delta_{1}, \Delta_{2}$ are the position shift of the first and second Gaussian distributions $\left(\Delta_{1}=-\Delta_{5}, \Delta_{2}=-\Delta_{4}\right), y_{1}, y_{2}, y_{3}$ are the height of the first, second and third Gaussian distributions $\left(y_{1}=y_{5}, y_{2}=y_{4}\right), \sigma_{1}, \sigma_{2}, \sigma_{3}$ are the width of the first, second and third Gaussian distributions $\left(\sigma_{1}=\sigma_{5}, \sigma_{2}=\sigma_{4}\right)$. It should be paid attention that the correlation nature between the yields of different mass number $\mathrm{A}$ is not symmetric, so it must take 15 parameters to calculate covariance matrix. In this case, the parameters $C$ and sensitivity matrix $F$ have 15 dimensions.

According to non-linear least square method and using iteration method, the optimal parameters were obtained:

$$
\begin{gathered}
\bar{C}=C^{(k+1)}=C^{k}+\left(F^{(k) T} V_{Y}^{-1} F^{(k)}\right)^{-1} F^{(k) T} V_{Y}^{-1}\left(Y-Y^{(K)}\right) \\
V_{\bar{C}}=\left(F^{(k) T} V_{Y}^{-1} F^{(k)}\right)^{-1}
\end{gathered}
$$


Table 1. The coefficients $a, b, c$ for each parameter.

\begin{tabular}{lcccccc}
\hline Coefficient & $\mathrm{a}$ & $\Delta \mathrm{a}$ & $\mathrm{b}$ & $\Delta \mathrm{b}$ & $\mathrm{c}$ & $\Delta \mathrm{c}$ \\
\hline$d_{1}$ & $2.2980 \mathrm{E}+01$ & $1.1719 \mathrm{E}-01$ & $-2.9532 \mathrm{E}-01$ & $4.7715 \mathrm{E}-02$ & $1.1423 \mathrm{E}-02$ & $4.7201 \mathrm{E}-03$ \\
$d_{2}$ & $1.6029 \mathrm{E}+01$ & $1.1547 \mathrm{E}-01$ & $-1.7469 \mathrm{E}-01$ & $4.8911 \mathrm{E}-02$ & $9.9681 \mathrm{E}-03$ & $4.4094 \mathrm{E}-03$ \\
$\mathrm{y}_{1}$ & $7.9992 \mathrm{E}+01$ & $1.5588 \mathrm{E}+00$ & $1.8373 \mathrm{E}+00$ & $5.8054 \mathrm{E}-01$ & $-9.9183 \mathrm{E}-02$ & $4.7307 \mathrm{E}-02$ \\
$\mathrm{y}_{2}$ & $1.9843 \mathrm{E}+01$ & $1.4994 \mathrm{E}+00$ & $-2.0535 \mathrm{E}+00$ & $5.4599 \mathrm{E}-01$ & $7.2597 \mathrm{E}-02$ & $4.2875 \mathrm{E}-02$ \\
$\mathrm{y}_{3}$ & $3.3165 \mathrm{E}-01$ & $1.3699 \mathrm{E}-01$ & $3.8917 \mathrm{E}-01$ & $8.4011 \mathrm{E}-02$ & $8.2379 \mathrm{E}-02$ & $1.0917 \mathrm{E}-02$ \\
$\sigma_{1}$ & $5.0553 \mathrm{E}+00$ & $4.6429 \mathrm{E}-02$ & $1.9431 \mathrm{E}-01$ & $2.0065 \mathrm{E}-02$ & $-4.9505 \mathrm{E}-03$ & $2.2101 \mathrm{E}-03$ \\
$\sigma_{2}$ & $2.8878 \mathrm{E}+00$ & $1.1557 \mathrm{E}-01$ & $2.3530 \mathrm{E}-02$ & $5.7062 \mathrm{E}-02$ & $-1.7867 \mathrm{E}-03$ & $6.1554 \mathrm{E}-03$ \\
$\sigma_{3}$ & $2.7694 \mathrm{E}+01$ & $1.5907 \mathrm{E}+00$ & $-2.6007 \mathrm{E}+00$ & $4.2610 \mathrm{E}-01$ & $8.8630 \mathrm{E}-02$ & $1.7585 \mathrm{E}-02$ \\
$\bar{A}$ & $1.1785 \mathrm{E}+02$ & $2.2110 \mathrm{E}-02$ & $7.4013 \mathrm{E}-02$ & $9.3442 \mathrm{E}-03$ & $-8.1667 \mathrm{E}-03$ & $8.7519 \mathrm{E}-04$ \\
\hline
\end{tabular}

$$
\begin{gathered}
\bar{Y}=Y^{(k+1)}=F^{(k)} C^{(k)}+Y^{(k)} \\
V_{\bar{Y}}=F^{(k)} V_{\bar{C}} F^{(k) T}
\end{gathered}
$$

where $F$ is the sensitivity matrix of the yield $Y$ to the parameters $C$. The initial values $Y^{(0)}$ were calculated according to the Wahl's formulas [11]. The iteration proceeds until it has converged. Because it is a problem of searching for optimal values for multi parameters, the solution is not unique. The parameters obtained must be chosen from the multi-set of possible solutions to keep the systematic behavior for each parameter at different energy points. This needs large amount of calculations and some times it is not easy.

The experimental data must be corrected and/or smoothed before they are fitted to insure the result is reasonable in physics and the iteration is convergent. In our practical situation, the mass distribution data, measured by double energy method, were corrected for mass resolution and smoothed with 7 points, and the chain yield data, measured by radioactive method were smoothed with 9 points due to the fact that the iteration is not convergent with 7 points.

Then the data were fitted by using 5 Gaussian model with iteration method for the mass distribution data and chain yield data at each energy point. The iteration is processed until it is convergent, the reduced $\chi^{2}$ is close to 1 and a set of reasonable parameters, called discrete parameters, is obtained. To search the systematic dependence of the parameters with the energy, the parameters were selected from multi sets of reasonable ones and were adjusted at the neighborhood of the parameter systematic trend sometimes.

\section{The systermatic of the parameters with energy}

Each parameter obtained at each energy point was fitted as energy function by least square method with a second order polynomial: $y=a+b x+c x^{2}$, whose coefficients $a, b, c$ were determined by a code to make the $\chi^{2}$ smaller. Each of the 9 parameters was fitted in the energy region from 0 to $20 \mathrm{MeV}$. The obtained coefficients $a, b$ and $c$ are given in table 1 and figures $1-4$, from which the parameters, called systematic parameters, can be calculated at any energy point.

The physical meaning can be seen clearly from the figures for the dependence of some parameters on energy. The parameter $a$, the mass number at the symmetric point of the double canal mass distribution, is decreased with the increasing

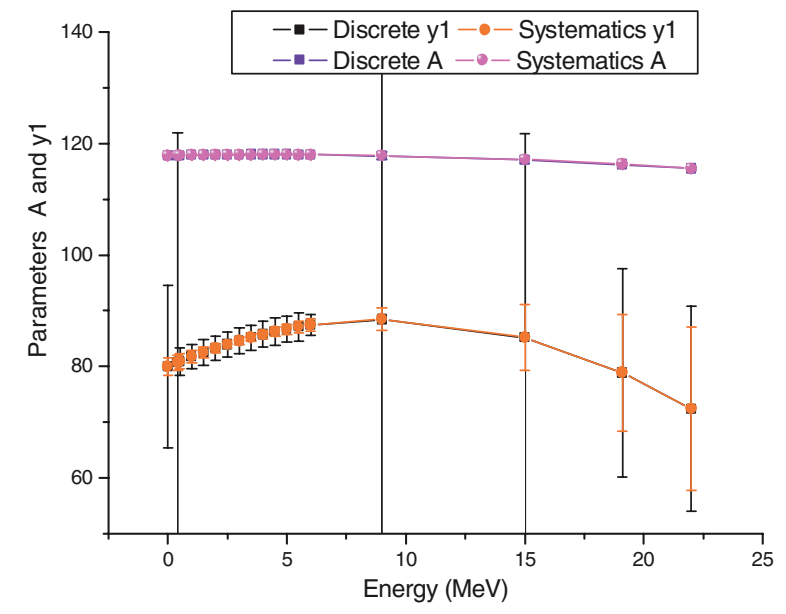

Fig. 1. Energy dependence of the systematic parameters $y_{1}, \bar{A}$.

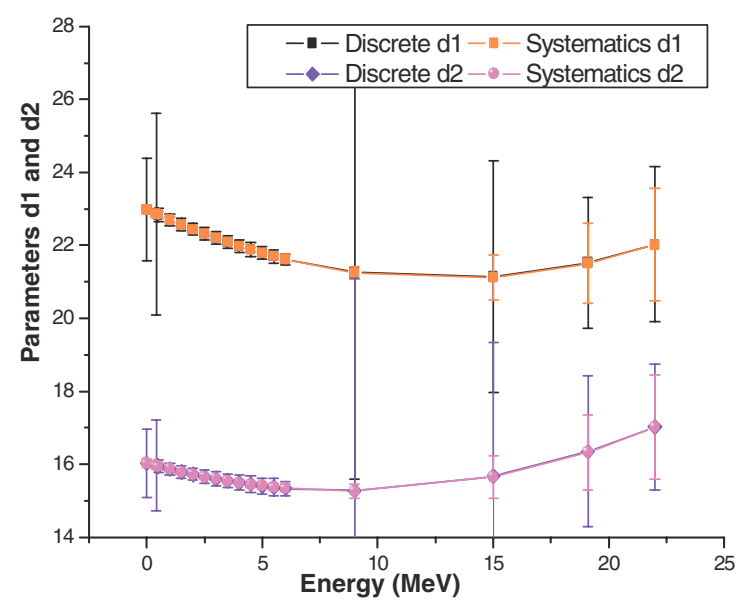

Fig. 2. Energy dependence of the systematic parameters $d_{1}, d_{2}$.

energy, which is due to that the fission $\bar{v}$ value is increased with increasing energy, so the total mass of the fission product nuclides is decreased. The parameter $y_{3}$, the height of the central Gaussian distribution, is increased with increasing energy, which describes the well known fact that the valley of the double canal mass distribution is increased with the energy increasing. The physical meaning of the other parameters can not be seen directly; due to the fact the calculated yield 


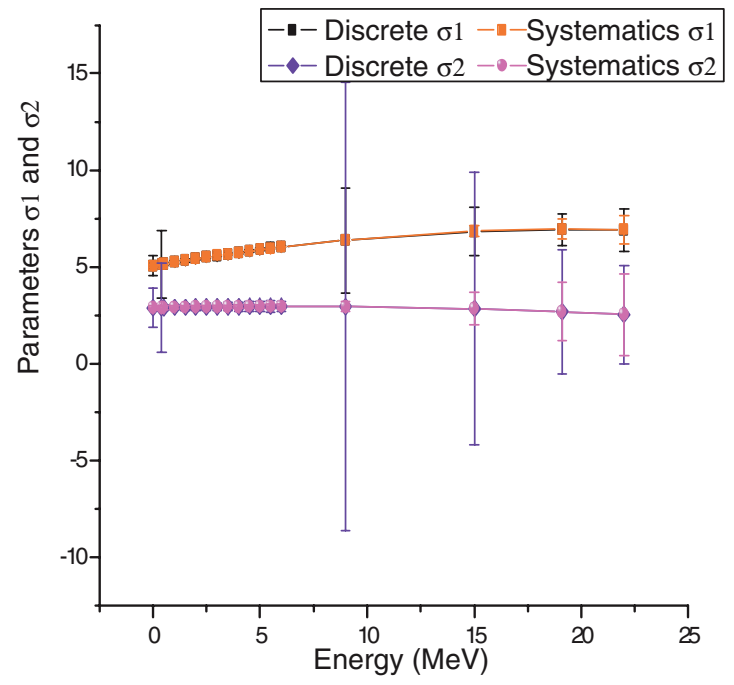

Fig. 3. Energy dependence of the systematic parameters $\sigma_{1}, \sigma_{2}$.

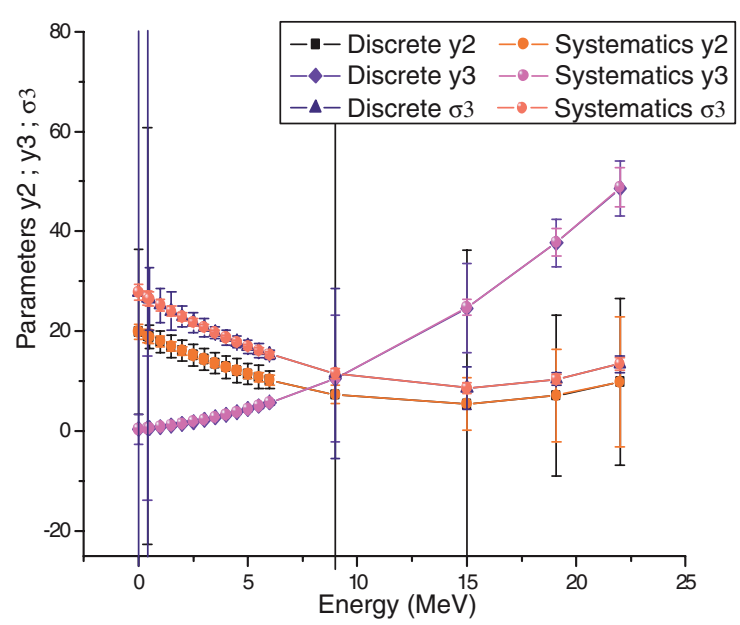

Fig. 4. Energy dependence of the systematic parameters $\mathrm{y}_{2}, \mathrm{y}_{3}$, and $\sigma_{3}$.

is a comprehensive result of 5 Gaussian, especially 2 pairs of symmetric Gaussian distribution contribution.

\section{The yield calculated with systematic}

By using the systematic parameters, the yield at any energy point can be calculated.

As examples, the comparison between the data calculated with systematic parameters and the experimental mass distribution data, measured with kinetic energy method (KEM) at 2.0 and $6.0 \mathrm{MeV}$ are given in figures 5-6 and the data with radio active method (RAM) at thermal energy and $15.0 \mathrm{MeV}$ are given in figures $7-8$. It can be seen that the systematic can reproduce the data measured with KEM well and the data measured with RAM roughly. The data measured by RAM are not complete for the mass A and also with some fluctuation. It seems that there is some systematical difference between the systematic and the data measured with RAM

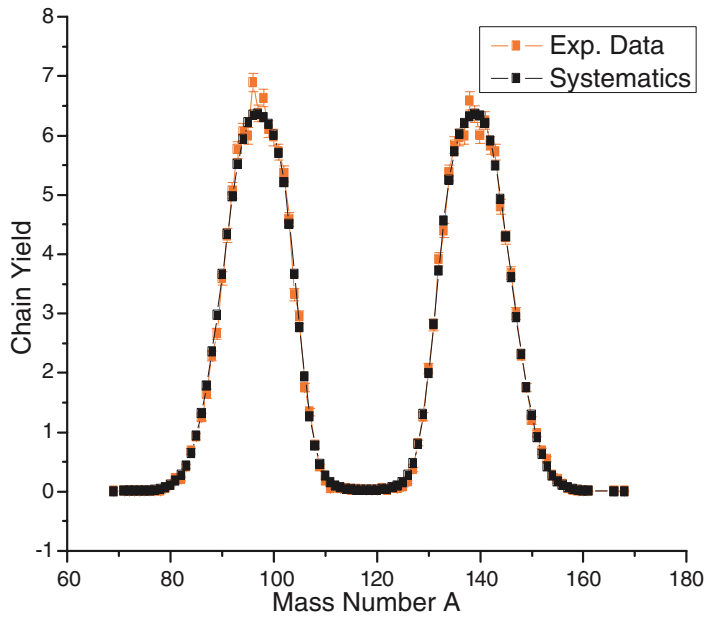

Fig. 5. Comparison of the yields calculated by the systematic with those measured by KEM at $2.0 \mathrm{MeV}$.

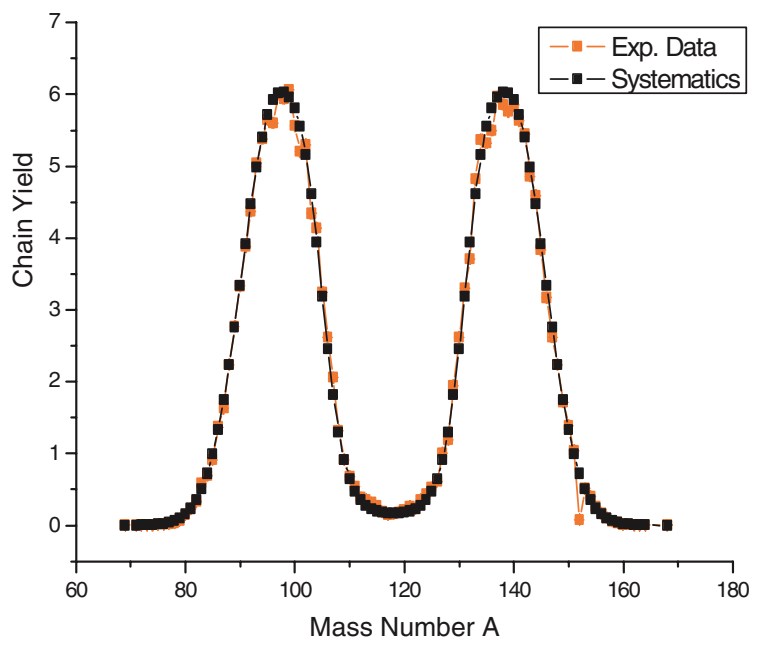

Fig. 6. Comparison of the yields calculated by the systematic with those measured by KEM at $6.0 \mathrm{MeV}$.

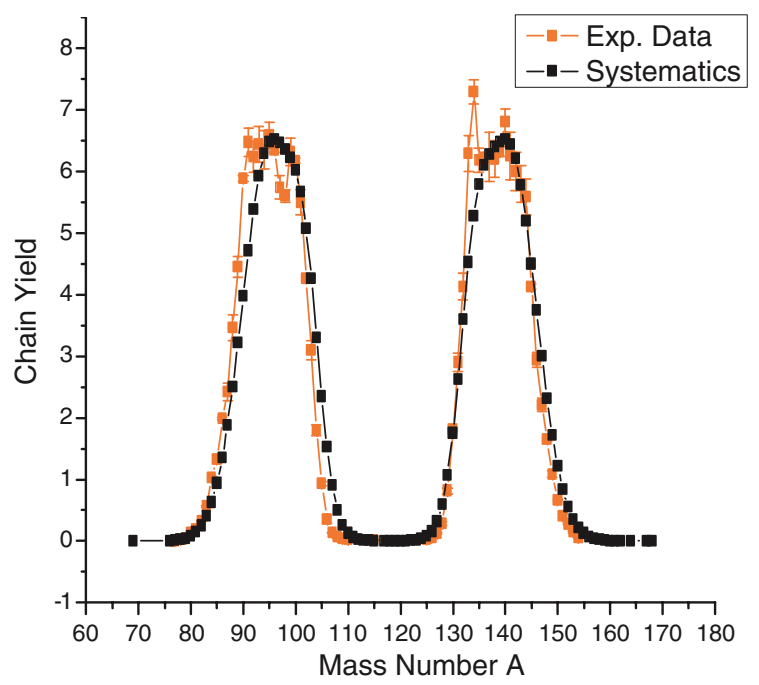

Fig. 7. Comparison of the yields calculated by the systematic with those measured by RAM at $0.0253 \mathrm{eV}$. 


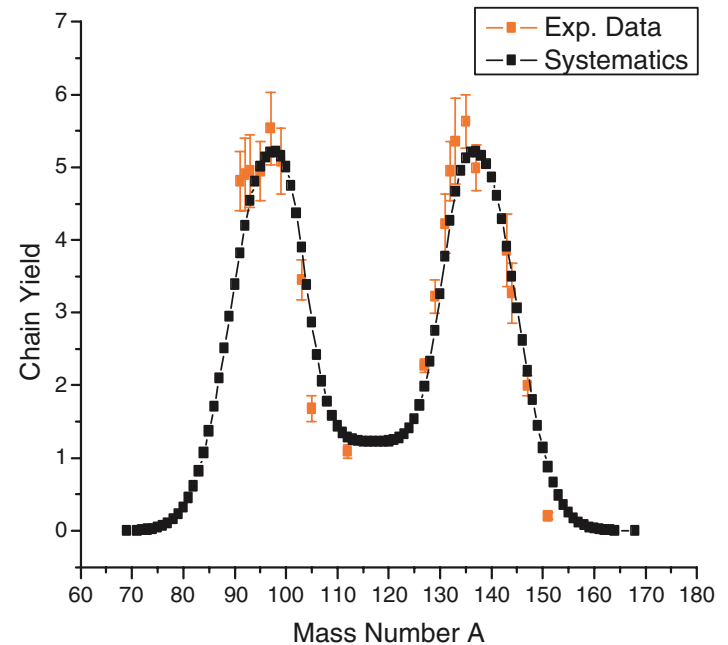

Fig. 8. Comparison of the yields calculated by the systematic with those measured by RAM at $15.0 \mathrm{MeV}$.

(see figs. 7-8), the data calculated with systematic are slightly shifted toward to the larger mass number A, especially for the distributions at low energies (fig. 7), which, substantively, reflects the systematical difference of the two kinds of experimental data. The similar matter was also found for ${ }^{238} \mathrm{U}$ [12]. The data measured with the KEM need to be corrected for mass resolution, neutron emission from fission compound and fragment, pulse height effect of the detector, fragment energy loss in the sample material, etc. These corrections are quite complicated and it is difficult to do them accurately. However, this kind of data must be used and as main base of the systematic, due to the fact the data are more complete and systematic for the incident neutron energy and fragment mass A.

\section{Conclusion remarks}

Based on the mass distribution data measured with KEM and RAM, the systematic on fission fragment mass distribution as a function of incident neutron energy was studied. The mass distribution data were fitted with 5 Gaussian model at each energy point and the discrete parameters were deduced. Each discrete parameter as function of energy was fitted with second order polynomials and the systematic parameters were deduced.

With the systematic, the yields of any mass A at any energy in the region from 0 to $20 \mathrm{MeV}$ can be calculated. The calculated results could reproduce the experimental data measured with KEM well, but show some systematical difference from the data measured by RAM, which reflects there is some systematical difference between the two kinds of measured data. The possible reason is that the data measured by KEM need more complicated accurate corrections.

\section{References}

1. FAN Tieshuan (2005) (private communication).

2. R.B. Strittmatter, ANS 27, 862 (1977).

3. G. Diiorio, NIM/B 147487 (1977).

4. G. Siegert, PR/C 14, 1864 (1976).

5. W.J. Maeck, 75WASH 1, 378 (1975).

6. J.W. Mandler, BAP 18, 768 (AB8) (1973).

7. Bao Jie et al., CNDP 26, 8 (2001).

8. Feng Jing et al., CNDP 25, 4 (2001).

9. Liu Tingjin.

10. Liu Tingjin, Data Correction to Mass Resolution, CNDP, 28, 2002.

11. A.C. Wahl, Systematic of Fission-Product Yields, RCM on Fission Yield, Vienna, Oct. 2001.

12. LIU Tingjin et al., Fission Yield Data Systematic for ${ }^{238} U$, Eng. Sci. 3(4), 50 (2005). 